\title{
Circulating matrix metalloproteinases and tissue metalloproteinase inhibitors in patients with idiopathic pulmonary fibrosis in the multicenter IPF-PRO Registry cohort
}

Jamie L. Todd ${ }^{1,2^{*}}$ (D), Richard Vinisko ${ }^{3}$, Yi Liu ${ }^{3}$, Megan L. Neely ${ }^{1,2}$, Robert Overton ${ }^{1}$, Kevin R. Flaherty ${ }^{4}$, Imre Noth ${ }^{5}$, L. Kristin Newby ${ }^{1,2,6}$, Joseph A. Lasky ${ }^{7}$, Mitchell A. Olman ${ }^{8}$, Christian Hesslinger ${ }^{9}$, Thomas B. Leonard ${ }^{3}$, Scott M. Palmer ${ }^{1,2+}$, John A. Belperio ${ }^{10 \dagger}$ and on behalf of the IPF-PRO Registry investigators

\begin{abstract}
Background: Matrix metalloproteinases (MMPs) and tissue inhibitors of MMPs (TIMPs) play important roles in the turnover of extracellular matrix and in the pathogenesis of idiopathic pulmonary fibrosis (IPF). This study aimed to determine the utility of circulating MMPs and TIMPs in distinguishing patients with IPF from controls and to explore associations between MMPs/TIMPs and measures of disease severity in patients with IPF.

Methods: The IPF cohort $(n=300)$ came from the IPF-PRO Registry, an observational multicenter registry of patients with IPF that was diagnosed or confirmed at the enrolling center in the past 6 months. Controls $(n=100)$ without known lung disease came from a population-based registry. Generalized linear models were used to compare circulating concentrations of MMPs 1, 2, 3, 7, 8, 9, 12, and 13 and TIMPs 1, 2, and 4 between patients with IPF and controls, and to investigate associations between circulating levels of these proteins and measures of IPF severity. Multivariable models were fit to identify the MMP/TIMPs that best distinguished patients with IPF from controls.

Results: All the MMP/TIMPs analyzed were present at significantly higher levels in patients with IPF compared with controls except for TIMP2. Multivariable analyses selected MMP8, MMP9 and TIMP1 as top candidates for distinguishing patients with IPF from controls. Higher concentrations of MMP7, MMP12, MMP13 and TIMP4 were significantly associated with lower diffusion capacity of the lung for carbon monoxide $\left(\mathrm{DL} \mathrm{L}_{\mathrm{co}}\right) \%$ predicted and higher composite physiologic index (worse disease). MMP9 was associated with the composite physiologic index. No MMP/TIMPs were associated with forced vital capacity \% predicted.
\end{abstract}

Conclusions: Circulating MMPs and TIMPs were broadly elevated among patients with IPF. Select MMP/TIMPs strongly associated with measures of disease severity. Our results identify potential MMP/TIMP targets for further development as disease-related biomarkers.

Keywords: Extracellular matrix, Biomarkers, Fibrosis, Interstitial lung diseases, Observational study

\footnotetext{
* Correspondence: jamie.todd@duke.edu

${ }^{+}$Scott M. Palmer and John A. Belperio contributed equally to this work.

'Duke Clinical Research Institute, Durham, NC, USA

2Division of Pulmonary, Allergy, and Critical Care Medicine, Department of

Internal Medicine, Duke University Medical Center, DUMC Box 103002,

Durham, NC 27710, USA

Full list of author information is available at the end of the article
}

(c) The Author(s). 2020 Open Access This article is licensed under a Creative Commons Attribution 4.0 International License, which permits use, sharing, adaptation, distribution and reproduction in any medium or format, as long as you give appropriate credit to the original author(s) and the source, provide a link to the Creative Commons licence, and indicate if changes were made. The images or other third party material in this article are included in the article's Creative Commons licence, unless indicated otherwise in a credit line to the material. If material is not included in the article's Creative Commons licence and your intended use is not permitted by statutory regulation or exceeds the permitted use, you will need to obtain permission directly from the copyright holder. To view a copy of this licence, visit http://creativecommons.org/licenses/by/4.0/ The Creative Commons Public Domain Dedication waiver (http://creativecommons.org/publicdomain/zero/1.0/) applies to the data made available in this article, unless otherwise stated in a credit line to the data. 


\section{Background}

Idiopathic pulmonary fibrosis (IPF) is a progressive interstitial lung disease associated with high mortality [1]. Two anti-fibrotic agents, nintedanib and pirfenidone, have been approved for the treatment of IPF and demonstrated to slow the progression of the disease $[2,3]$. However, the diagnosis and management of IPF remain challenging, with no clinically available biomarkers to serve as adjuncts in diagnosis or prediction of prognosis or treatment response.

The pathobiology of IPF involves excess production of extracellular matrix (ECM) and dysregulated matrix remodeling [4]. Matrix metalloproteinases (MMPs) are a family of zinc-dependent endopeptidases important in ECM degradation. Expression of MMPs and their physiological inhibitors, tissue inhibitors of MMPs (TIMPs), is tightly regulated in the lung, with notable upregulation during lung development, tissue injury, and host defense [5].

Several basic and clinical studies have underscored the importance of MMPs and TIMPs in the pathobiology of IPF, as recently reviewed [6]. In particular, murine models of bleomycin-induced pulmonary fibrosis demonstrated increased expression of MMPs and TIMPs, while mice with genetic deletions in select MMPs had reduced lung fibrosis after bleomycin administration compared with wild type mice [6, 7]. Patients with IPF showed increased MMP and TIMP expression in the lungs [8-10], including in structural cells (for example, the epithelium) and immune cells (for example, interstitial macrophages) [8]. Circulating levels of MMPs 1, 3, 7, 8 , and 9 have been shown to be elevated in patients with IPF $[9,11]$ and higher circulating levels of MMP7 to be associated with more severe disease [11], a higher risk of disease worsening over a 3-year period [12], and shorter survival time [13]. However, there remains a relative paucity of information on the full range of MMPs and TIMPs detectable in the blood of patients with IPF and their utility as biomarkers. We sought to determine expression of MMPs 1, 2, 3, 7, 8, 9, 12, and 13 and TIMPs 1,2 , and 4 in a cohort of well-characterized patients with IPF, to understand if combinations of MMPs and TIMPs could distinguish patients with IPF from controls, and to investigate associations between MMPs/ TIMPs and measures of IPF severity.

\section{Methods}

\section{IPF cohort}

The IPF cohort was drawn from the multicenter observational US Idiopathic Pulmonary Fibrosis Prospective Outcomes (IPF-PRO) Registry (NCT01915511) [14] that enrolled patients with IPF that was diagnosed or confirmed at the enrolling center in the past 6 months. The cohort for this analysis consisted of 300 patients enrolled by 1 February 2017, who had an enrollment blood sample, data on critical clinical variables at enrollment, and an assessment form indicating the certainty of the IPF diagnosis (definite, probable, possible) determined by the investigator according to the 2011 American Thoracic Society/European Respiratory Society/Japanese Respiratory Society/Latin American Thoracic Association (ATS/ ERS/JRS/ALAT) diagnostic guidelines [15].

\section{Control cohort}

The control cohort was drawn from the Measurement to Understand the Reclassification of Disease of Cabarrus/Kannapolis (MURDOCK) Study, a registry of adult residents of North Carolina in which self-reported health information and biological samples are collected [16]. To ensure that the control cohort had a similar age, race and ethnicity distribution to the IPF cohort, participants considered for inclusion as controls were White, nonHispanic and aged 60 to 80 years. Participants were excluded if they had self-reported respiratory disease, cancer, or autoimmune disease at enrollment or during follow-up, were active smokers, had active second-hand tobacco exposure, or reported use of respiratorytargeted medications or immunomodulators. Random sampling with stratification by sex and smoking status (ever versus never) was used to select 100 controls with a similar distribution of these characteristics to the IPF cohort.

\section{MMP and TIMP quantification}

Enrollment plasma samples were assayed for antigenic levels of MMPs 1, 2, 3, 7, 8, 9, 12, and 13 and TIMPs 1 , 2, and 4 (ThermoFisher; Vienna, Austria). MMPs 2, 3, 9 and TIMP1 and MMPs $1,7,8,12$, and 13 were quantified using multiplexed luminex immunoassays. TIMP2 and TIMP4 were quantified by ELISA. Samples that fell below the standard curve for MMP1 ( $n=23$ IPF and $n=$ 17 control), MMP8 ( $n=48$ IPF and $n=63$ control), MMP12 ( $n=7$ IPF and $n=4$ control), and MMP13 $(n=$ $10 \mathrm{IPF}$ and $n=3$ control) were extrapolated if feasible or assigned a concentration of half the minimum observed value. No samples fell below the standard curve for MMPs 2, 3, 7, or 9 or TIMPs 1, 2, and 4 .

\section{Statistical analyses}

All statistical analyses were completed in SAS version 9.4 or $\mathrm{R}$ version 3.5.1. Generalized linear modeling was used to compare MMP and TIMP concentrations between patients with IPF and controls. The data were $\log 10$ transformed to more closely meet the distribution assumptions for linear models. Descriptive box-plots were generated from the $\log 10$ transformed data. The results of the statistical analyses were back-transformed to the original scale and described as geometric means 
and geometric mean ratios of the IPF versus control groups. Correction for multiple comparisons was performed using the Benjamini-Hochberg method to control the false discovery rate at $5 \%$.

Multivariable analyses were performed to assess whether a set of MMPs or TIMPs could differentiate patients with IPF from controls, and $\log 10$ data were centered, scaled, and Box-Cox transformed to improve model efficiency. No MMPs or TIMPs were highly correlated (Pearson correlation coefficient of $\geq 0.9$ ) so all analytes were retained in the analysis. The data from the 400-patient cohort were randomly divided into training $(n=300 ; 75 \%)$ and test sets ( $n=100 ; 25 \%$ ) using stratified sampling to retain the 3:1 ratio in each set. Three linear models (penalized logistic regression, linear discriminant analysis, partial least squares) and 4 non-linear models (K-nearest neighbors, support vector machines, recursive partitioning [single tree], random forests [boosted trees]) were fit. Covariates for age and sex were included in each model. When fitting each model, 10fold cross validation was used to choose the optimal tuning parameter based on the area under the receiver operating curve. Operating characteristics (accuracy, sensitivity, specificity, area under the receiver operating curve) were determined in the training set and then evaluated in the test set. Variable importance measures were determined for the best performing models [17]. The model with the best performance based on the area under the curve (AUC) was also refit using the full 400-patient cohort.

For univariate analyses in the IPF cohort, linear regression models were employed on the $\log 10$ transformed data to determine the association between circulating levels of each MMP/TIMP and three measures of disease severity, analyzed as continuous variables: FVC \% predicted, $\mathrm{DL}_{\mathrm{CO}} \%$ predicted, and the composite physiologic index (CPI), which correlates with the extent of fibrosis on radiography in patients with IPF [18]. The National Health and Nutrition Examination Survey (NHANES) III reference eqs [19]. were used to calculate the \% predicted values for FVC and FEV1, forced expiratory volume in $1 \mathrm{~s}$ $\left(\mathrm{FEV}_{1}\right)$, and the reference equations developed by Crapo and Morris [20] were used to calculate the \% predicted values for DLco. The linear regression analyses were repeated adjusting for treatment (nintedanib, pirfenidone, neither) at enrollment. The Benjamini-Hochberg method was used to control the false discovery rate at $5 \%$. For each disease severity measure, the estimated coefficients and confidence intervals from the linear regression model were used to calculate the estimated difference in the disease severity measure between the median MMP/TIMP concentration in patients in the lowest tertile of disease severity and the median concentration in patients in the highest tertile of disease severity.
In multivariable analyses of the IPF cohort, FVC \% predicted, $\mathrm{DL}_{\mathrm{CO}} \%$ predicted, and $\mathrm{CPI}$ were modeled separately to examine their relationship with sets of MMPs/TIMPs. Pairwise correlation analysis indicated that no MMPs or TIMPs were highly correlated (Pearson correlation coefficient of $\geq 0.9$ ) so all were retained in the analysis. The performance of 2 linear models (partial least squares and penalized linear regression) and 4 non-linear models (K-nearest neighbors, support vector machines, recursive partitioning [single tree], random forests [boosted trees]) was measured on training ( $n=$ $225 ; 75 \%)$ and test $(n=75 ; 25 \%)$ sets. Covariates for age, sex, current use of nintedanib, and current use of pirfenidone were included in each model. While fitting each model, 10-fold cross-validation was used to choose the optimal tuning parameter in the training set, minimizing the root mean squared error (RMSE). R-squared was computed.

\section{Results \\ Cohort characteristics}

In the control cohort $(n=100)$, the median $(\mathrm{Q} 1, \mathrm{Q} 3)$ age was $66.0(63.0,71.5)$ years, $74 \%$ were men, $68 \%$ were former smokers (Table 1$)$. In the IPF cohort $(n=300)$, the median (Q1, Q3) age was $70.0(65.0,75.0)$ years, $74 \%$ were men, $94 \%$ were white and $67 \%$ were former smokers (Table 1). Most patients with IPF (73\%) were characterized as having definite IPF; $10.3 \%$ were determined by the enrolling physician to have clinically significant emphysema on CT scan and 54\% were taking nintedanib or pirfenidone. Median (Q1, Q3) FVC \% predicted was $69.7(61.0,80.2), \mathrm{DL}_{\mathrm{CO}} \%$ predicted was 40.6 $(31.7,49.4)$ and CPI was $53.5(46.6,60.5)$. The lower and upper tertile cutpoints for these measures are shown in Additional file 1.

\section{Associations between MMP/TIMP levels and presence or severity of IPF}

The level of each MMP and TIMP analyzed was significantly higher (corrected $p$-value $<0.05$ ) in patients with IPF versus controls with the exception of TIMP2 (Fig. 1, Table 2, Additional file 2). The highest geometric mean ratios of concentration in patients with IPF versus controls were observed with MMP8 (4.05), MMP1 (2.11) and MMP9 (2.07) (Table 2).

The median (Q1, Q3) concentrations of each MMP/ TIMP by tertile of FVC $\%$ predicted, $\mathrm{DL}_{\mathrm{CO}} \%$ predicted, and CPI in the IPF cohort are described in Additional file 3. In linear regression analyses, there were no statistically significant associations between levels of MMPs or TIMPs and FVC \% predicted. However, higher levels of MMPs 7, 8, 12, and 13 and TIMP4 were significantly associated with lower $\mathrm{DL}_{\mathrm{CO}} \%$ predicted and higher (worse) CPI score (Table 3). Additionally, higher 
Table 1 Characteristics of the IPF and control cohorts

\begin{tabular}{|c|c|c|}
\hline Characteristic & $\operatorname{IPF}(N=300)$ & Control $(N=100)$ \\
\hline Age (years) & $70.0(65.0,75.0)$ & $66.0(63.0,71.5)$ \\
\hline Male & $223(74.3 \%)$ & $74(74 \%)$ \\
\hline \multicolumn{3}{|l|}{ Race } \\
\hline White & $281(93.7 \%)$ & $100(100 \%)$ \\
\hline Black or African-American & $8(2.7 \%)$ & $0(0 \%)$ \\
\hline Asian & $6(2.0 \%)$ & $0(0 \%)$ \\
\hline Other & $5(1.7 \%)$ & $0(0 \%)$ \\
\hline Ethnicity: Hispanic or Latino & $8(2.7 \%)$ & $0(0 \%)$ \\
\hline \multicolumn{3}{|l|}{ Smoking } \\
\hline Past & $202(67.3 \%)$ & $68(68 \%)$ \\
\hline Never & $96(32.0 \%)$ & $32(32 \%)$ \\
\hline Current & $2(0.7 \%)$ & $0(0 \%)$ \\
\hline \multicolumn{3}{|l|}{ Diagnostic criteria $^{a}$} \\
\hline Definite IPF & $220(73.3 \%)$ & - \\
\hline Probable IPF & $63(21.0 \%)$ & - \\
\hline Possible IPF & $17(5.7 \%)$ & - \\
\hline Presence of emphysema on CT & $31(10.3 \%)$ & - \\
\hline Supplemental oxygen use at rest & $59(20.0 \%)^{b}$ & - \\
\hline \multicolumn{3}{|l|}{ Pulmonary function measures } \\
\hline $\mathrm{FEV}_{1}(\mathrm{~L})$ & $2.2(1.8,2.7)$ & - \\
\hline $\mathrm{FEV}_{1}(\%$ predicted $)$ & $77.4(68.0,89.1)$ & - \\
\hline FVC (L) & $2.7(2.2,3.2)$ & - \\
\hline FVC (\% predicted) & $69.7(61.0,80.2)$ & - \\
\hline $\mathrm{FEV}_{1} / \mathrm{FVC}$ ratio & $74.1(72.8,89.6)$ & - \\
\hline $\mathrm{DL}_{\mathrm{CO}}(\mathrm{mL} / \mathrm{min} / \mathrm{kPa})$ & $12.0(8.6,14.7)$ & - \\
\hline $\mathrm{DL}_{\mathrm{CO}}$ (\% predicted) & $40.6(31.7,49.4)$ & - \\
\hline CPI & $53.5(46.6,60.5)$ & - \\
\hline \multicolumn{3}{|l|}{ Antifibrotic drug use } \\
\hline Nintedanib & $56(18.7 \%)$ & $0(0 \%)$ \\
\hline Pirfenidone & $106(35.3 \%)$ & $0(0 \%)$ \\
\hline Neither & $138(46.0 \%)$ & 100 (100\%) \\
\hline
\end{tabular}

Values are median (Q1, Q3) or $\mathrm{n}(\%)$

$C T$ computed tomography, $C P I$ composite physiologic index, $D L_{C O}$ diffusing capacity of the lungs for carbon monoxide, $F E V_{1}$ forced expiratory volume in 1 s, FVC forced vital capacity

a Determined by the investigator according to the 2011 ATS/ERS/JRS/ALAT diagnostic guidelines [15]

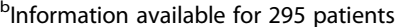

levels of MMP9 were significantly associated with higher CPI (Table 3). MMP7 had the largest estimated effect size in both the $\mathrm{DL}_{\mathrm{CO}}$ and CPI analyses, followed by TIMP4 (DL $\mathrm{DO}_{\mathrm{CO}}$ and $\left.\mathrm{CPI}\right)$ and MMP13 $\left(\mathrm{DL}_{\mathrm{CO}}\right)$. Specifically, the estimated differences in $\mathrm{DL}_{\mathrm{CO}} \%$ predicted per 10-fold increase in circulating MMP7, TIMP4, and MMP13 were $-13.41,-11.80 \%$, and $-6.37 \%$, respectively. For CPI, the estimated difference per 10-fold increase in circulating MMP7 was 11.01 points, while for
TIMP4 it was 7.94 points (Table 3). Adjusting these regression analyses for anti-fibrotic treatment did not influence the significance nor the effect estimates of the association between these proteins and disease severity (Additional file 4).

Given that prior reports suggest that circulating MMP or TIMP expression can be altered in smoking-related respiratory diseases such as chronic obstructive pulmonary disease and emphysema [21, 22], and a substantial proportion of the patients with IPF and controls in our study were former smokers, we investigated whether there were differences in MMP or TIMP expression by current/past versus never smoking status. No significant differences were observed in either the IPF or control populations (Additional file 5). We also compared MMP and TIMP concentrations between subjects with vs. without clinically significant emphysema on CT scan. These analyses demonstrated that circulating MMP2 was significantly higher among patients with clinically significant radiographic emphysema (Additional file 6). None of the other measured MMPs or TIMPS were statistically different between these two groups. Additionally, among the IPF cohort, none of the MMP/TIMPs analyzed significantly associated with $\mathrm{FEV}_{1} \%$ predicted.

\section{Sets of MMPs/TIMPs that best discriminate IPF}

As nearly all of the MMPs and TIMPs measured were elevated in patients with IPF, we sought to understand whether sets of these proteins differentiated patients with IPF from controls better than any single protein. There was no collinearity for the MMPs and TIMPs, thus a multivariable approach may more appropriately account for synergistic or antagonistic relationships that may exist between these molecules. We found that in general, linear multivariable models (penalized logistic regression, partial least squares, and linear discriminant analysis) had similar or better operating characteristics compared with more complex non-linear methods in the training set, with most models obtaining classification accuracies between 80 and $90 \%$ over all iterations of the cross-validation procedure (Fig. 2). In the training set, penalized logistic regression was the best performing linear model with an AUC of 0.89 (SD 0.04), while random forests was the best performing non-linear model (AUC 0.90 [SD 0.05]) (Fig. 2). In the test set, based on the AUC, the penalized logistic regression model also performed the best, with a classification accuracy of 0.89 (Fig. 3, Table 4). Variable importance measures were determined for the three linear models and the best performing nonlinear model (random forests). TIMP1, MMP9 and MMP8 were the top proteins of importance across the models (Fig. 4). Next, the penalized logistic regression model was refit to all the data (test and training sets). A histogram of the regression scores for each 


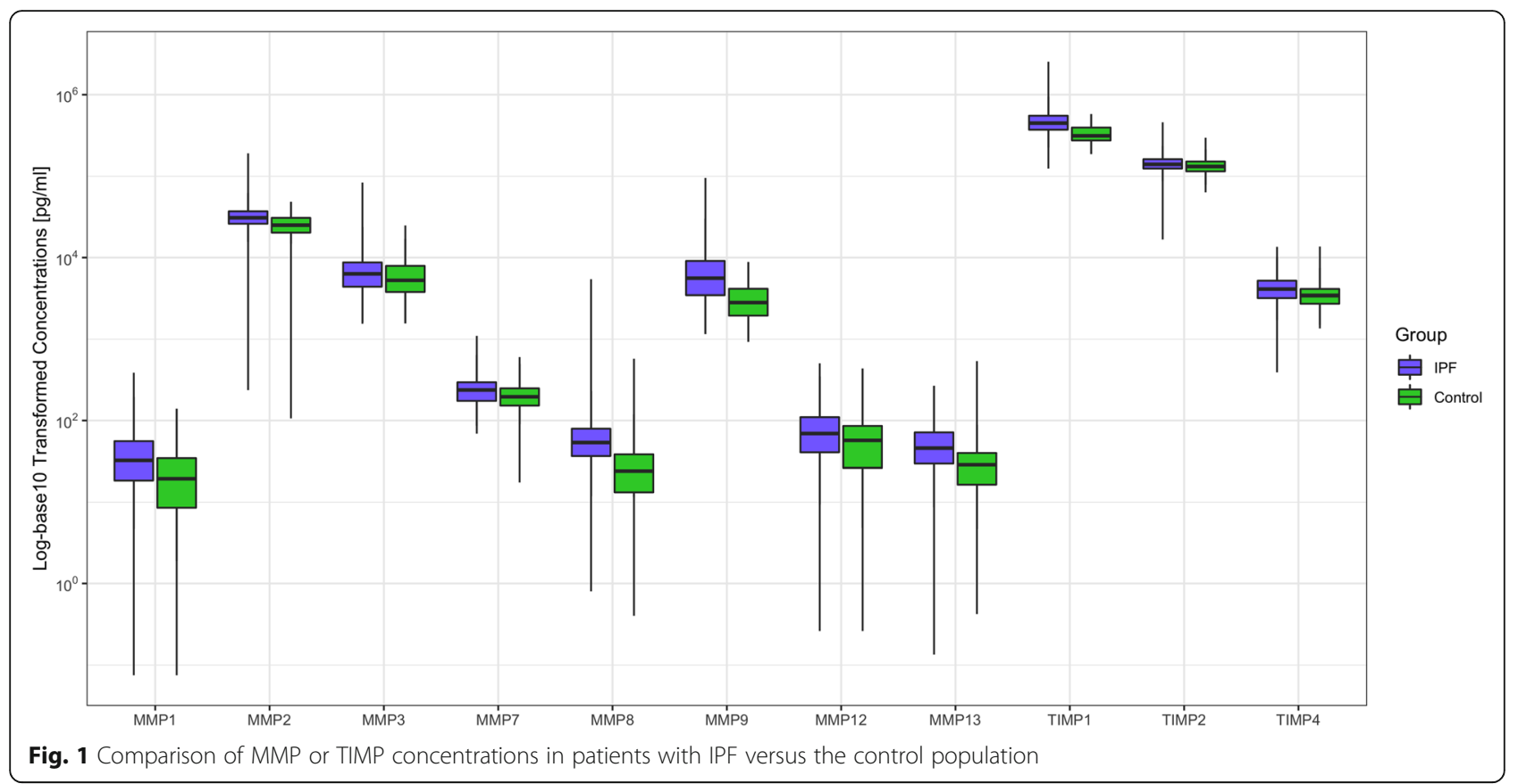

Table 2 Associations between MMPs/TIMPs and IPF versus control status, ordered by corrected $p$-value

\begin{tabular}{|c|c|c|c|c|}
\hline Protein $(\mathrm{pg} / \mathrm{mL})$ & Group & gMean & RatiolPF:control $\%^{\mathrm{a}}$ & Corrected $p$-value \\
\hline \multirow[t]{2}{*}{ MMP8 } & Control & 13.68 & & \\
\hline & IPF & 55.41 & 4.05 & $<.0001$ \\
\hline \multirow[t]{2}{*}{ MMP9 } & Control & 2801.98 & & \\
\hline & IPF & 5793.87 & 2.07 & $<.0001$ \\
\hline \multirow[t]{2}{*}{ TIMP1 } & Control & $326,688.87$ & & \\
\hline & IPF & $456,465.47$ & 1.40 & $<.0001$ \\
\hline \multirow[t]{2}{*}{ MMP1 } & Control & 11.77 & & \\
\hline & IPF & 24.88 & 2.11 & $<.0001$ \\
\hline \multirow[t]{2}{*}{ TIMP4 } & Control & 3414.36 & & \\
\hline & IPF & 4067.08 & 1.19 & 0.0004 \\
\hline \multirow[t]{2}{*}{ MMP7 } & Control & 196.39 & & \\
\hline & IPF & 234.88 & 1.20 & 0.0008 \\
\hline \multirow[t]{2}{*}{ MMP2 } & Control & $22,709.02$ & & \\
\hline & IPF & $29,111.36$ & 1.28 & 0.0024 \\
\hline \multirow[t]{2}{*}{ MMP13 } & Control & 25.20 & & \\
\hline & IPF & 37.55 & 1.49 & 0.0024 \\
\hline \multirow[t]{2}{*}{ MMP12 } & Control & 41.85 & & \\
\hline & IPF & 58.16 & 1.39 & 0.0097 \\
\hline \multirow[t]{2}{*}{ MMP3 } & Control & 5393.62 & & \\
\hline & $\mathrm{IPF}$ & 6370.79 & 1.18 & 0.0164 \\
\hline \multirow[t]{2}{*}{ TIMP2 } & Control & $134,417.53$ & & \\
\hline & IPF & $141,289.58$ & 1.05 & 0.1029 \\
\hline
\end{tabular}

${ }^{a}$ Represents the ratio of the geometric mean concentration for each protein in patients with IPF relative to controls. Model includes IPF status (yes/no) as factor ${ }^{b} p$-values were corrected for multiple comparisons using the Benjamini-Hochberg method to control the false discovery rate at $5 \%$ 
Table 3 Association between MMPs/TIMPs and clinical measures of IPF severity

\begin{tabular}{|c|c|c|c|c|c|c|c|c|c|}
\hline \multirow[t]{2}{*}{ Protein } & \multicolumn{3}{|c|}{ Association with FVC \% predicted } & \multicolumn{3}{|c|}{ Association with $\mathrm{DLco} \%$ predicted } & \multicolumn{3}{|c|}{ Association with CPI } \\
\hline & $\begin{array}{l}\text { Estimated effect } \\
(\beta)^{\mathbf{a}}\end{array}$ & $\begin{array}{l}\text { Estimated } \\
\text { effect }^{\mathbf{b}}\end{array}$ & $\begin{array}{l}\text { Corrected } \\
\boldsymbol{p} \text {-value }\end{array}$ & $\begin{array}{l}\text { Estimated effect } \\
(\beta)^{\mathbf{a}}\end{array}$ & $\begin{array}{l}\text { Estimated } \\
\text { effect }^{\mathbf{b}}\end{array}$ & $\begin{array}{l}\text { Corrected } \\
\boldsymbol{p} \text {-value }\end{array}$ & $\begin{array}{l}\text { Estimated effect } \\
(\beta)^{\mathbf{a}}\end{array}$ & $\begin{array}{l}\text { Estimated } \\
\text { effect }^{\mathbf{b}}\end{array}$ & $\begin{array}{l}\text { Corrected } \\
\boldsymbol{p} \text {-value }\end{array}$ \\
\hline MMP1 & -0.72 & -0.0747 & 0.7547 & -1.82 & -0.1717 & 0.2488 & 1.28 & 0.1325 & 0.3279 \\
\hline MMP2 & 4.93 & 0.0391 & 0.2859 & -1.27 & -0.0284 & 0.7314 & 0.03 & 0.0006 & 0.9886 \\
\hline MMP3 & 2.35 & 0.0729 & 0.7547 & -3.94 & -0.1193 & 0.2700 & 2.26 & 0.0430 & 0.4260 \\
\hline MMP7 & -10.22 & -0.4649 & 0.1616 & -13.41 & -1.0998 & 0.0074 & 11.01 & 0.8748 & 0.0056 \\
\hline MMP8 & -6.34 & -0.2639 & 0.1616 & -5.90 & -0.6893 & 0.0409 & 5.47 & 0.5862 & 0.0151 \\
\hline MMP9 & -7.96 & -1.2797 & 0.1518 & -3.87 & -0.6113 & 0.2488 & 5.34 & 1.0360 & 0.0286 \\
\hline MMP12 & -3.90 & -0.4665 & 0.1871 & -5.54 & -0.5672 & 0.0074 & 4.37 & 0.4930 & 0.0073 \\
\hline MMP13 & -2.98 & -0.2180 & 0.2503 & -6.37 & -0.8935 & 0.0005 & 4.70 & 0.6179 & 0.0016 \\
\hline TIMP1 & -1.06 & -0.0007 & 0.8715 & 0.57 & 0.0195 & 0.9173 & 1.15 & 0.0242 & 0.8708 \\
\hline TIMP2 & -3.36 & -0.0026 & 0.7547 & -6.75 & -0.0237 & 0.4094 & 5.49 & 0.0379 & 0.4260 \\
\hline TIMP4 & -2.66 & -0.0059 & 0.7547 & -11.80 & -0.9541 & 0.0261 & 7.94 & 0.6367 & 0.0497 \\
\hline
\end{tabular}

${ }^{a}$ Estimated difference in disease severity measure per 10-fold increase in protein concentration, as determined by the linear regression model

${ }^{\mathrm{b}}$ The estimated linear regression coefficients (B) and confidence intervals were used to calculate the estimated difference in the disease severity measure going from the median of tertile 1 to the median of tertile 3 in MMP or TIMP concentration

${ }^{c} p$-value determined by linear regression corrected for multiplicity using the Benjamini-Hochberg method to control the false discovery rate at $5 \%$

subject, calculated using the model coefficients for MMP8 (- 0.55), MMP9 (-0.65) and TIMP1 (-0.64) and their respective concentrations, is plotted in Fig. 5, with lower scores associating with IPF.

\section{Prediction of IPF disease severity using MMP and TIMP measurements}

Multivariable models were fit to understand whether sets of MMPs/TIMPs predicted measures of disease severity in this IPF cohort. The maximum R-squared value achieved for predicting any disease severity measure was 0.16 in the training set (Additional file 7). R-squared values were lower in the test set (Additional file 8). These results indicate that no enhancement in discrimination of disease severity was achieved when considering sets of MMPs/TIMPs.

\section{Discussion}

We present the first study to quantify expression of a broad array of circulating MMPs and TIMPs in a

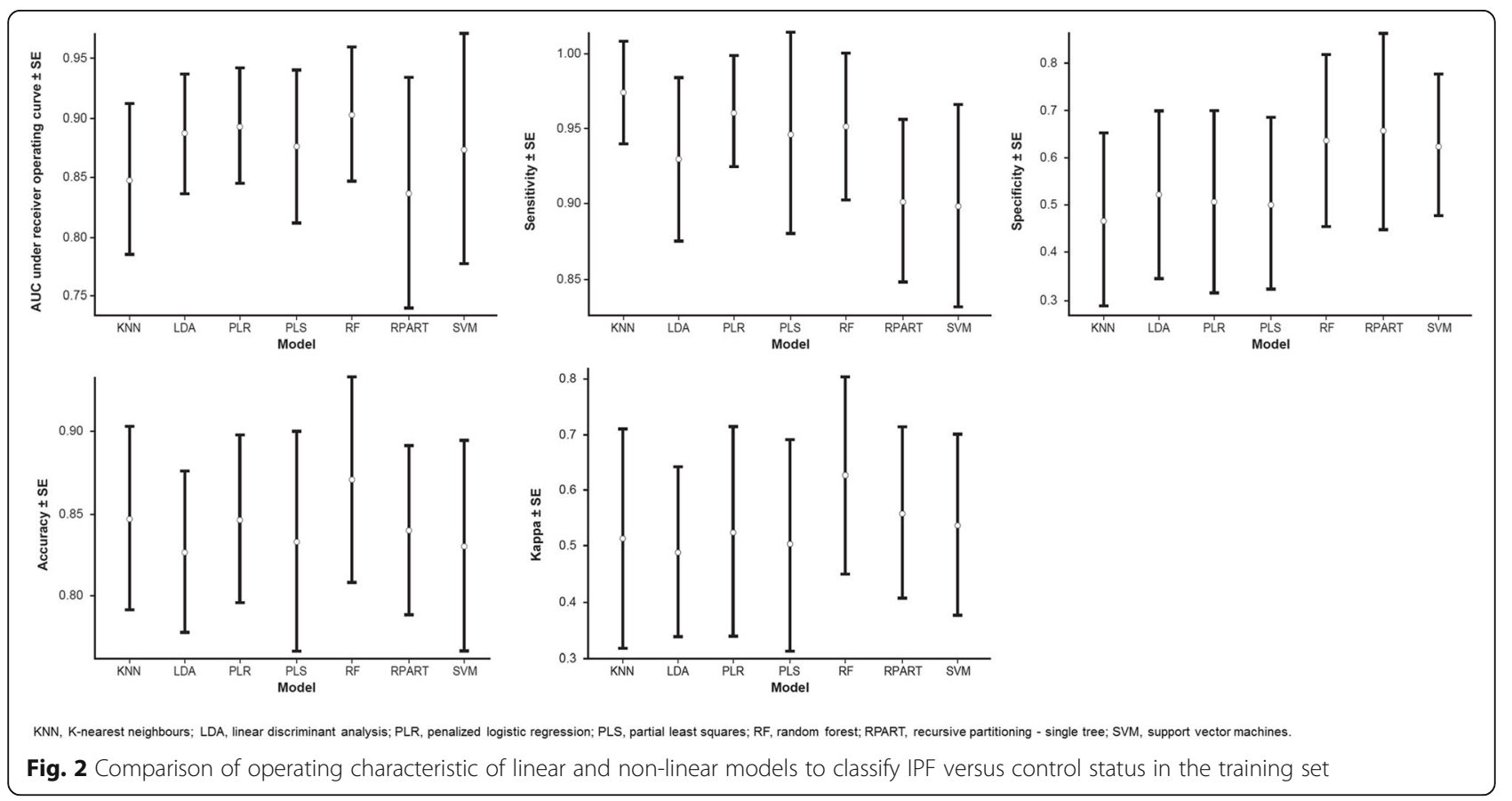




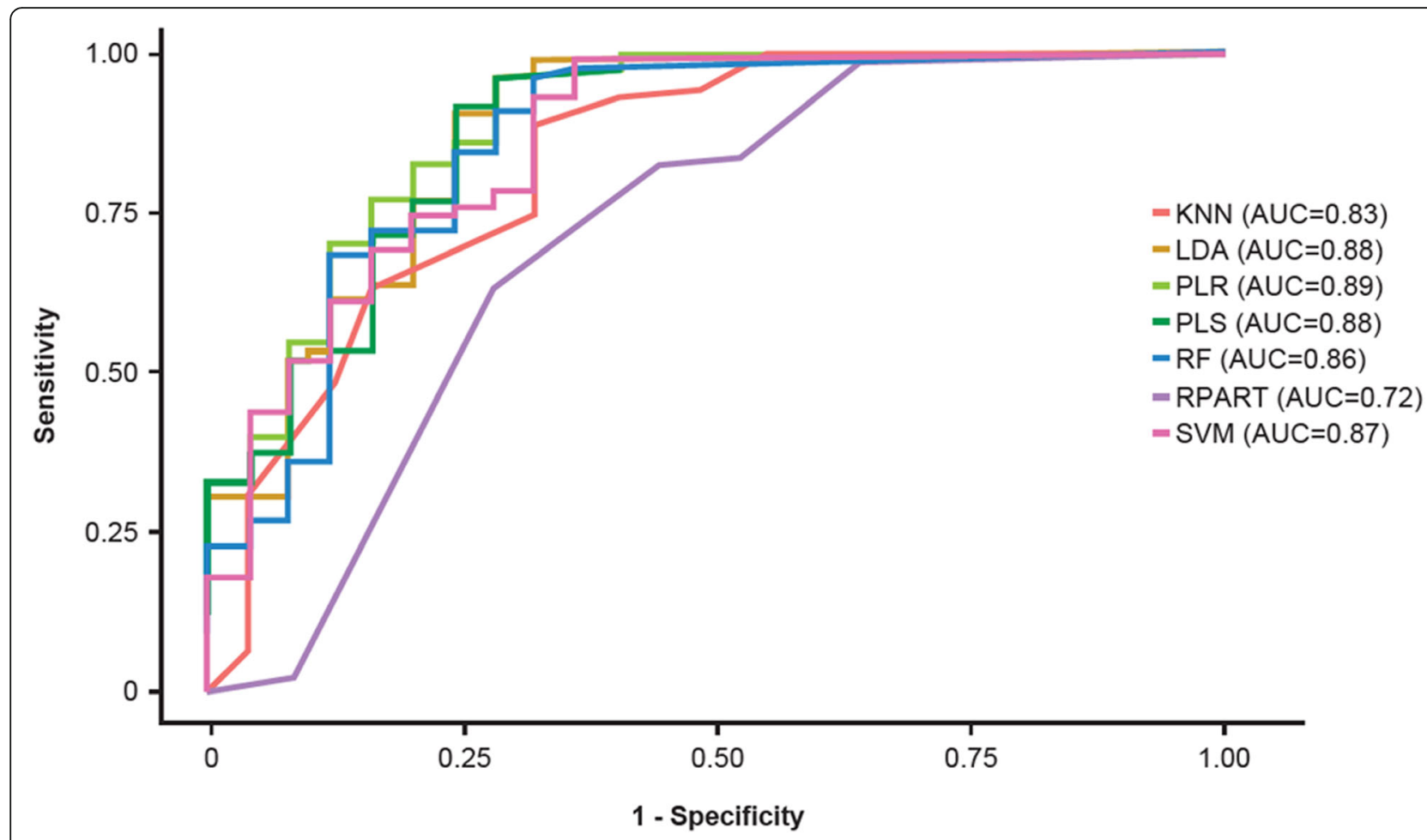

KNN, K-nearest neighbours; LDA, linear discriminant analysis; PLR, penalized logistic regression; PLS, partial least squares; RF, random forest; RPART, recursive partitioning - single tree; SVM, support vector machines.

Fig. 3 Receiver operating curves for the test data fit across linear and non-linear multivariable models

multicenter cohort of well-characterized patients with IPF and in controls without known lung disease. This study not only provides insights into how single MMPs/TIMPs relate to IPF status and severity, but also considers the influence of combinations of these proteins. Our results demonstrate that circulating MMPs and TIMPs are generally elevated among patients with IPF, consistent with altered extracellular matrix remodeling. The combination of MMPs 8 and 9 and TIMP1 demonstrated good performance characteristics in differentiating patients with IPF from controls with a similar distribution of age, sex, and

Table 4 Operating characteristic of linear and non-linear models to classify IPF versus control status in the test set

\begin{tabular}{llllll}
\hline Model & AUC & Sensitivity & Specificity & Accuracy & Kappa \\
\hline PLS & 0.88 & 0.99 & 0.60 & 0.89 & 0.67 \\
PLR & 0.89 & 0.99 & 0.60 & 0.89 & 0.67 \\
LDA & 0.88 & 0.99 & 0.64 & 0.90 & 0.70 \\
SVM & 0.87 & 0.93 & 0.64 & 0.86 & 0.61 \\
KNN & 0.83 & 0.95 & 0.52 & 0.84 & 0.52 \\
RPART & 0.72 & 0.84 & 0.48 & 0.75 & 0.32 \\
RF & 0.87 & 0.96 & 0.64 & 0.88 & 0.65 \\
\hline
\end{tabular}

AUC area under the curve, KNN K-nearest neighbors, LDA linear discriminant analysis, $P L R$ penalized logistic regression, $P L S$ partial least squares, $R F$ random forests, RPART recursive partitioning; SVM, support vector machines smoking status. Moreover, several MMPs, in addition to TIMP4, strongly associated with CPI. The association between MMPs/TIMPs and CPI appeared to be driven mostly by $\mathrm{DL}_{\mathrm{CO}} \%$ predicted, as no MMP/TIMP significantly associated with FVC \% predicted.

In our analyses, circulating levels of MMPs 7, 8, 12, and 13 associated with both $\mathrm{DL}_{\mathrm{CO}}$ and $\mathrm{CPI}$, while MMP9 associated with CPI only. Though we found MMP2 concentrations to be higher among IPF patients with clinically significant radiographic emphysema, we did not find a significant association between MMP2 and $\mathrm{DL}_{\mathrm{CO}}$. MMP7 had the largest estimated effect size in our disease severity analyses. Prior work has shown that MMP7 is elevated in patients with IPF [11, 12, 23$25]$ and is negatively correlated with $\mathrm{DL}_{\mathrm{CO}}[11,24]$. Previous studies have also suggested that circulating MMP7 concentrations increase as FVC \% predicted values decline [12] and that elevated MMP7 concentrations may identify patients with IPF with a worse prognosis [13, 23]. However, a recent study found that while MMP7 was elevated in patients with IPF compared with controls, there was no difference in baseline concentrations of MMP7 between patients whose disease progressed or did not progress over a 52-week follow-up period [25].

In addition to supporting the value of MMP7 as a marker of IPF severity, our work identified other MMPs 


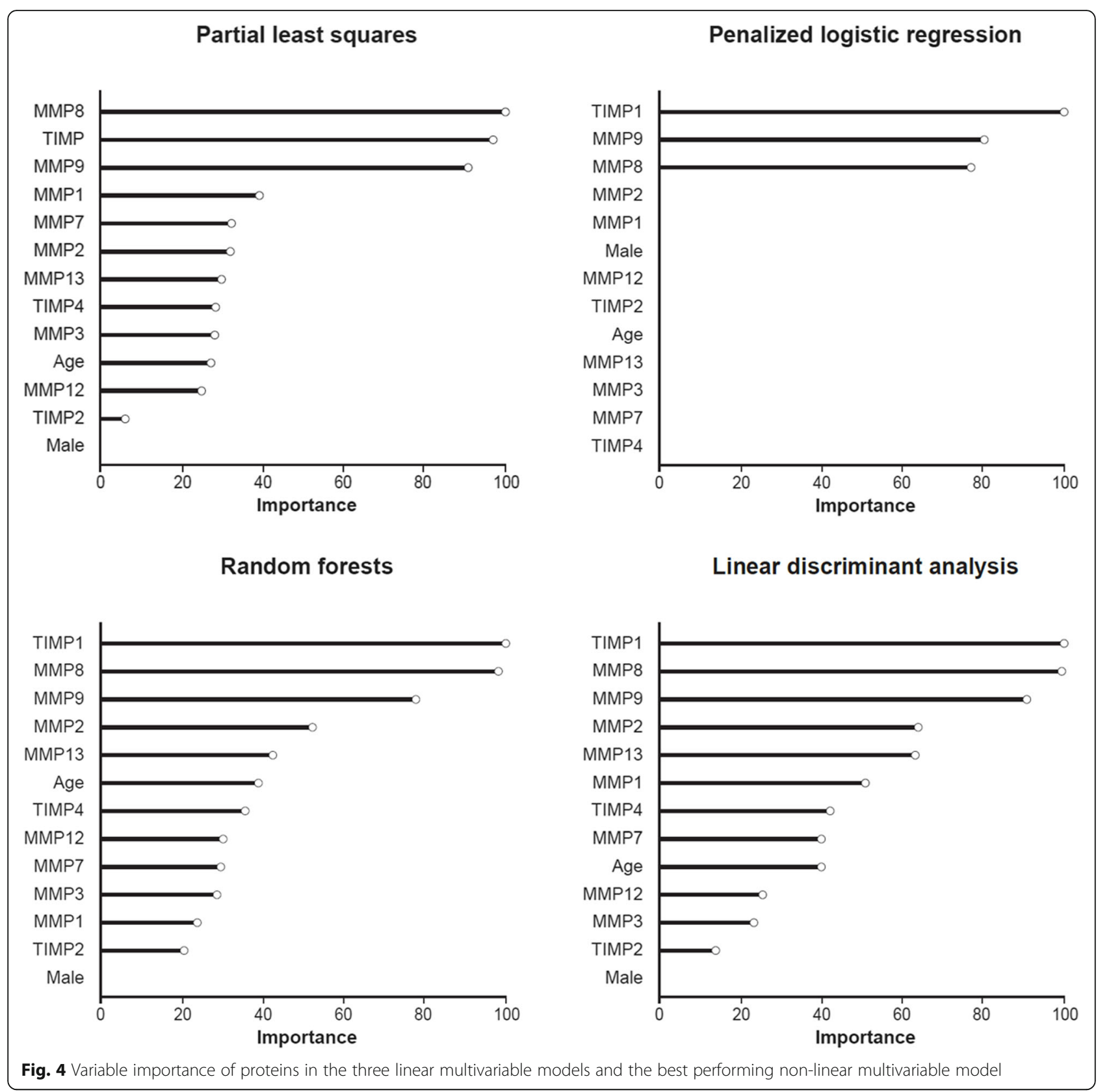

associated with disease severity measures. Previous studies have shown that circulating levels of MMP8 are increased in patients with IPF $[9,11]$, although they did not correlate with disease severity measures, including $\mathrm{DL}_{\mathrm{CO}}$, in a cohort of 74 patients [9]. Increased circulating levels of MMP3 and MMP9 in patients with IPF have also been reported, although their association with pulmonary function was not investigated [11]. We found that circulating concentrations of MMPs 12 and 13 were increased in patients with IPF and associated with $\mathrm{DL}_{\mathrm{CO}}$ and CPI. Studies in murine models of pulmonary fibrosis have yielded inconsistent results regarding the roles of these MMPs after exposure to bleomycin, radiation, or other insults [26-31]. Few clinical data exist regarding circulating levels of these MMPs, but MMP13 has been shown to be overexpressed in the lungs of patients with IPF [30]. Among patients with systemic sclerosis, MMP12 concentrations were increased in patients who had interstitial lung disease compared with those who did not, and correlated with the degree of pulmonary restriction [32]. Together, these observations support further investigation into the potential value of MMPs 12 and 13 to predict clinically relevant outcomes among patients with IPF.

In our IPF cohort, we observed higher levels of TIMP4 to be associated with lower $\mathrm{DL}_{\mathrm{CO}}$ and higher (worse) 


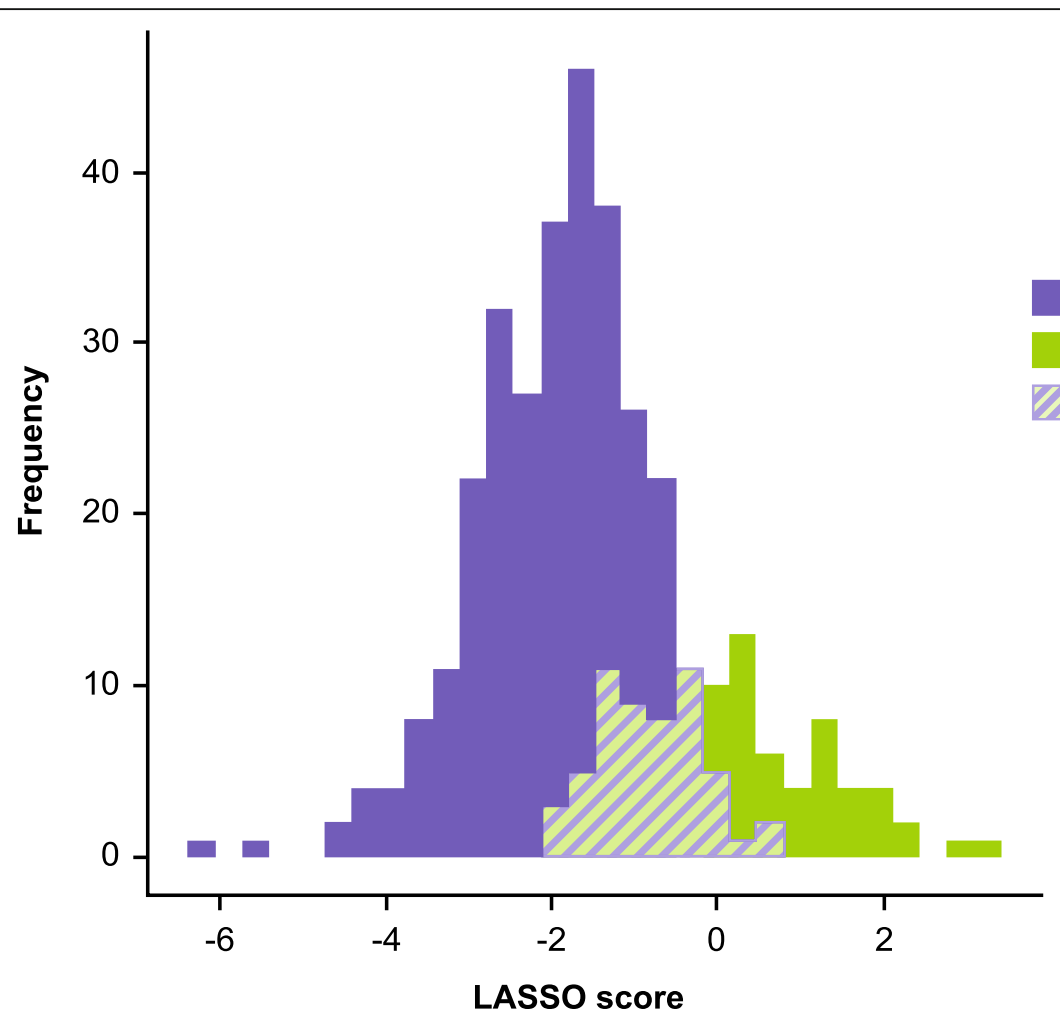

Fig. 5 Histogram of the penalized logistic regression model scores for each subject in the IPF and control cohorts

CPI. This finding is of interest, as TIMP4 differs from other TIMPs in that at homeostasis, its expression is restricted to cardiovascular structures [33]. Moreover, studies in individuals with pulmonary hypertension have indicated that TIMP4 levels correlate with hemodynamic parameters [34]. It is unknown whether the association we observed between $\mathrm{DL}_{\mathrm{CO}}$ or $\mathrm{CPI}$ and peripheral blood TIMP4 expression reflects pulmonary vascular remodeling and development of pulmonary hypertension, accumulating lung fibrosis, or the effects of angiogenesis supporting pulmonary hypertension and fibroplasia.

Consistent with our results, a prior study implicated MMP8 as a differentiating protein in a 5-protein classifier (MMP1, MMP7, MMP8, IGFBP-1, TNFRS1A) of IPF status, although the classification model did not select MMP9 and did not consider TIMP1 [11]. Our results suggest that the combination of MMP8, MMP9 and TIMP1 provides good discriminatory ability in classifying individuals as having or not having IPF, with TIMP1 being the most important distinguishing variable in two of the best performing models. Collectively, these studies support the role of evaluating multiple MMP/ TIMP sets to aid in determining the presence and severity of IPF. Additionally, such data may provide useful guidance for future investigations, such as those focused on MMP/TIMPs that discriminate IPF from other fibrosing lung diseases or those examining patients with early interstitial lung abnormalities of uncertain clinical significance.

When our data on circulating MMP or TIMP levels in patients with IPF are considered in the context of data from experimental models of fibrosis, a clearer view emerges of the potential mechanism(s) by which altered MMP/TIMP expression contributes to lung fibrosis. Both MMP3 and MMP7 appear critical to the development of experimental lung fibrosis, with rodents genetically deficient in MMP3 or MMP7 demonstrating a reduction in pulmonary fibrosis after bleomycin challenge [35-37]. MMP7 can promote pulmonary neutrophil recruitment and chemokine dependent angiogenesis, two important processes in the development of lung fibroplasia [37-42]. The pro-fibrotic nature of MMPs 3 and MMP7 is supported by our observation that higher levels of these MMPs are associated with higher CPI, i.e. more severe disease, among patients with IPF. MMP12 has been found to be pro-fibrotic during bleomycin-induced lung fibrosis [27, 43-45], in line with our findings that elevated levels of MMP12 are associated with worse CPI.

In contrast to these pro-fibrotic MMPs, animal models suggest that augmented levels of MMP1 decrease fibroplasia in liver, muscle and heart [46-48], while MMP2 decreased type I collagen production in experimental liver fibrosis [49]. This could imply that the elevations in circulating MMP1 and MMP2 expression we observed 
in patients with IPF are a failed attempt to control lung fibrosis. MMP13 cleaves and so reduces the activity of CCL2 and CXCL12 [29, 50]. This suggests that augmented levels of MMP13 may be anti-fibrotic by decreasing the recruitment of CCR2-expressing "profibrotic" macrophages [51] and CCR2- and CXCR4expressing fibrocytes $[52,53]$. TIMP1-/- mice have not been shown to develop reduced lung fibrosis in response to bleomycin [31, 54]; however, our finding of augmented levels of human TIMP1 suggests an unsuccessful attempt at regulating fibrosis in IPF. Collectively, our human data, in conjunction with data from animal models, insinuate that pro-fibrotic mediators (MMP3, MMP7, and particularly MMP12) overwhelm any antifibrotic effects mediated by MMP1, MMP2, MMP13, and TIMP1, leading to increased extracellular matrix deposition and impairments in pulmonary function and gas exchange.

While our study has several strengths, including the multicenter nature of the IPF cohort, we acknowledge that it has inherent limitations. First, although we characterized a broad array of MMPs, we did not include all described MMPs nor TIMP3. Additionally, while our MMP assay provides precise quantification of circulating MMP concentrations, MMP activity and organ specificity cannot be inferred. Second, as pulmonary function data were not available for the majority of subjects prior to the date of enrolment, we cannot ascertain whether patients were experiencing significant disease progression or were relatively stable at the time of sampling. Finally, although this work contributes important new information regarding circulating MMP and TIMP expression in patients with IPF, our study was not designed to understand whether the observed changes are specific to IPF as compared with non-IPF fibrosing lung diseases. As the IPF-PRO Registry has recently expanded to include patients with non-IPF interstitial lung diseases in the ILD-PRO Registry, we anticipate that future studies will address the specificity of candidate biomarkers for IPF as compared with other fibrosing lung diseases.

\section{Conclusion}

The results of this study further delineate the potential value of selected MMPs or TIMPs as disease-related biomarkers in patients with IPF. Further validation will be necessary, as will extension of these analyses to examine associations between MMP/TIMP expression and clinical outcomes. Rich longitudinal data collected in the IPF-PRO Registry, including serial pulmonary function measures, hospitalization data, and information on vital status will support these analyses and further the goal of improving the diagnosis and management of patients with IPF.

\section{Supplementary information}

Supplementary information accompanies this paper at https://doi.org/10. 1186/s12890-020-1103-4.

Additional file 1: Tertile cutpoints for disease severity metrics.

Additional file 2: MMP and TIMP concentrations $(\mathrm{pg} / \mathrm{mL})$ in patients with IPF versus the control population.

Additional file 3: Median (first quartile, third quartile) concentration for each MMP/TIMP stratified by tertile of FVC \% predicted, DLCO \% predicted, and CPI.

Additional file 4: Association of MMPs and TIMPs with measures of IPF severity, adjusted for anti-fibrotic treatment.

Additional file 5: Mean (standard deviation) log 10 MMP and TIMP concentrations in the IPF and control populations as stratified by smoking status at enrollment (current/past smoker vs. never smoker).

Additional file 6: Mean (standard deviation) $\log 10$ MMP and TIMP concentrations in patients with IPF with or without clinically significant emphysema on CT scan as assessed by the enrolling physician.

Additional file 7: Model performance in the training (cross-validation) set for baseline FVC \% predicted, DLCO \% predicted and CPI.

Additional file 8: Model performance in the test set for baseline FVC \% predicted, DLCo \% predicted and CPI.

\section{Acknowledgements}

The authors acknowledge the IPF-PRO Registry participants and principal investigators: Wael Asi, Renovatio Clinical, The Woodlands, TX; Albert Baker, Lynchburg Pulmonary Associates, Lynchburg, VA; Scott Beegle, Albany Medical Center, Albany, NY; John A. Belperio, University of California Los Angeles, Los Angeles, CA; Rany Condos, NYU Medical Center, New York, NY; Francis Cordova, Temple University, Philadelphia, PA; Daniel A. Culver, Cleveland Clinic, Cleveland, OH; Tracey Luckhardt (formerly Joao A.M. de Andrade), University of Alabama at Birmingham, Birmingham, AL; Daniel Dilling, Loyola University Health System, Maywood, IL; Kevin R. Flaherty, University of Michigan, Ann Arbor, Ml; Marilyn Glassberg, University of Miami, Miami, FL; Mridu Gulati, Yale School of Medicine, New Haven, CT; Kalpalatha Guntupalli, Baylor College of Medicine, Houston, TX; Nishant Gupta, University of Cincinnati Medical Center, Cincinnati, $\mathrm{OH}$; Amy Hajari Case, Piedmont Healthcare, Austell, GA; David Hotchkin, The Oregon Clinic, Portland, OR; Tristan Huie, National Jewish Hospital, Denver, CO; Robert Kaner, Weill Cornell Medical College, New York, NY; Hyun Kim, University of Minnesota, Minneapolis, MN; Maryl Kreider, University of Pennsylvania, Philadelphia, PA; Lisa Lancaster, Vanderbilt University, Nashville, TN; Joseph Lasky, Tulane University, New Orleans, LA; David Lederer, Columbia University Medical Center/New York Presbyterian Hospital, New York, NY; Doug Lee, Wilmington Health and PMG Research, Wilmington, NC; Timothy Liesching, Lahey Clinic, Burlington, MA; Randolph Lipchik, Froedtert \& The Medical College of Wisconsin Community Physicians, Milwaukee, Wl; Jason Lobo, UNC Chapel Hill, Chapel Hill, NC; Yolanda Mageto, Baylor University Medical Center at Dallas, Dallas, TX; Prema Menon, Vermont Lung Center, Colchester, VT; Lake Morrison, Duke University Medical Center, Durham, NC; Andrew Namen, Wake Forest University, Winston Salem, NC; Justin Oldham, University of California, Davis, Sacramento, CA; Rishi Raj, Stanford University, Stanford, CA; Murali Ramaswamy, Pulmonlx LLC, Greensboro, NC; Tonya Russell, Washington University, St. Louis, MO; Paul Sachs, Pulmonary Associates of Stamford, Stamford, CT; Zeenat Safdar, Houston Methodist Lung Center, Houston, TX; Barry Sigal, Salem Chest and Southeastern Clinical Research Center, Winston Salem, NC; Leann Silhan, UT Southwestern Medical Center, Dallas, TX; Mary Strek, University of Chicago, Chicago, IL; Sally Suliman, University of Louisville, Louisville, KY; Jeremy Tabak, South Miami Hospital, South Miami, FL; Rajat Walia, St. Joseph's Hospital, Phoenix, AZ; Timothy P. Whelan, Medical University of South Carolina, Charleston, SC. The authors acknowledge the contribution of Katey Durham, formerly of Boehringer Ingelheim Pharmaceuticals, Inc., to this work.

Writing support was provided by Elizabeth $\mathrm{Ng}$ and Wendy Morris of FleishmanHillard Fishburn, London, UK, which was contracted and funded by Boehringer Ingelheim Pharmaceuticals, Inc. The authors meet criteria for authorship as recommended by the International Committee of Medical Journal Editors (ICMJE). Boehringer Ingelheim was given the opportunity to 
review the manuscript for medical and scientific accuracy as well as intellectual property considerations.

\section{Authors' contributions}

All authors contributed to the design of the study; $J L T, J L, M A O, L K N, K R F, T L$, $\mathrm{CH}, \mathrm{IN}, J \mathrm{AB}$, and SMP contributed to the acquisition of the data; MLN, RO, $K D, Y L$, and RV contributed to the development of the analysis plan and RV, $\mathrm{KD}$ and $\mathrm{YL}$ performed the data analysis. All authors contributed to the interpretation of the data. JLT drafted the manuscript. All authors critically revised the manuscript and approved the final version for submission.

\section{Funding}

The IPF-PRO Registry is funded by Boehringer Ingelheim Pharmaceuticals, Inc. and coordinated by the Duke Clinical Research Institute. This study also used biosample resources acquired through the MURDOCK Study Community Registry and Biorepository, which acknowledges funding from the David H. Murdock Institute for Business \& Culture and Duke University's CTSA grant (UL1TR001117) from the National Institutes of Health's National Center for Advancing Translational Sciences. Boehringer Ingelheim Pharmaceuticals, Inc. was involved in the design of this study and the writing of this manuscript.

\section{Availability of data and materials}

A summary of data generated or analyzed during this study are included in this published article or its supplementary information files.

\section{Ethics approval and consent to participate}

The IPF-PRO Registry study obtained ethics approval at the data coordinating center (Duke Clinical Research Institute, Duke Institutional Review Board Protocol Number Pro00046131) and at every enrolling center (listed in the Acknowledgments). All participants gave written informed consent. Additionally, ethics approval was granted by the Duke Institutional Review Board Protocol Number Pro00082241 to use the biosamples obtained as part of the IPF-PRO Registry for the analyses contained herein.

\section{Consent for publication}

Not applicable.

\section{Competing interests}

$J L T, M L N, R O, L K N$ and SMP are employees of the Duke Clinical Research Institute, which receives funding support from Boehringer Ingelheim Pharmaceuticals, Inc. to coordinate the IPF-PRO Registry. KRF reports grants and personal fees from Boehringer Ingelheim and Roche/Genentech and personal fees from FibroGen, Sanofi Genzyme, and Veracyte. IN reports personal fees from Boehringer Ingelheim, Genentech and ImmuneWorks. JAL reports personal fees from Boehringer Ingelheim, Roche/Genentech, Galecto, Biogen and Veracyte. MAO reports research grant and grant review fees from Boehringer Ingelheim. RV, KD, YL, CH and TBL are employees of Boehringer Ingelheim. JAB has no competing interests.

\section{Author details}

'Duke Clinical Research Institute, Durham, NC, USA. ²Division of Pulmonary, Allergy, and Critical Care Medicine, Department of Internal Medicine, Duke University Medical Center, DUMC Box 103002, Durham, NC 27710, USA. ${ }^{3}$ Boehringer Ingelheim Pharmaceuticals Inc., Ridgefield, CT, USA. ${ }^{4}$ Division of Pulmonary and Critical Care Medicine, University of Michigan, Ann Arbor, MI, USA. ${ }^{5}$ University of Virginia, Charlottesville, VA, USA. ${ }^{6}$ Duke Clinical \& Translational Science Institute, Durham, NC, USA. ${ }^{7}$ School of Medicine, Tulane University, New Orleans, LA, USA. ${ }^{8}$ Department of Inflammation and Immunity and Respiratory Institute, Cleveland Clinic, Cleveland, OH, USA. ${ }^{9}$ Boehringer Ingelheim Pharma GmbH \& Co. KG, Biberach, Germany. ${ }^{10}$ David Geffen School of Medicine at UCLA, Los Angeles, CA, USA.

\section{Received: 29 July 2019 Accepted: 28 February 2020}

Published online: 14 March 2020

\section{References}

1. Raghu G, Remy-Jardin M, Myers JL, Richeldi L, Ryerson CJ, Lederer DJ, et al. Diagnosis of idiopathic pulmonary fibrosis. An official ATS/ERS/JRS/ALAT clinical practice guideline. Am J Respir Crit Care Med. 2018;198(5):e44-68.
2. King TE Jr, Bradford WZ, Castro-Bernardini S, Fagan EA, Glaspole I, Glassberg MK, et al. A phase 3 trial of pirfenidone in patients with idiopathic pulmonary fibrosis. N Engl J Med. 2014;370(22):2083-92.

3. Richeldi L, du Bois RM, Raghu G, Azuma A, Brown KK, Costabel U, et al. Efficacy and safety of nintedanib in idiopathic pulmonary fibrosis. N Engl J Med. 2014;370(22):2071-82

4. Selman M, King TE, Pardo A. Idiopathic pulmonary fibrosis: prevailing and evolving hypotheses about its pathogenesis and implications for therapy. Ann Intern Med. 2001;134:136-51.

5. Parks WC, Shapiro SD. Matrix metalloproteinases in lung biology. Respir Res. 2001;2:10-9

6. Pardo A, Cabrera S, Maldonado M, Selman M. Role of matrix metalloproteinases in the pathogenesis of idiopathic pulmonary fibrosis. Respir Res. 2016;17:23.

7. Madtes DK, Elston AL, Kaback LA, Clark JG. Selective induction of tissue inhibitor of metalloproteinase-1 in bleomycin-induced pulmonary fibrosis. Am J Respir Cell Mol Biol. 2001;24(5):599-607.

8. Selman M, Ruiz V, Cabrera S, Segura L, Ramírez R, Barrios R, et al. TIMP-1, -2, -3 , and -4 in idiopathic pulmonary fibrosis. A prevailing nondegradative lung microenvironment? Am J Physiol Lung Cell Mol Physiol. 2000;279(3): L562-74.

9. Craig VJ, Polverino F, Laucho-Contreras ME, Shi Y, Liu Y, Osorio JC, et al. Mononuclear phagocytes and airway epithelial cells: novel sources of matrix metalloproteinase-8 (MMP-8) in patients with idiopathic pulmonary fibrosis. PLoS One. 2014;9(5):e97485.

10. DePianto DJ, Chandriani S, Abbas AR, Jia G, N'Diaye EN, Caplazi P, et al. Heterogeneous gene expression signatures correspond to distinct lung pathologies and biomarkers of disease severity in idiopathic pulmonary fibrosis. Thorax. 2015;70(1):48-56.

11. Rosas IO, Richards TJ, Konishi K, Zhang Y, Gibson K, Lokshin AE, et al. MMP1 and MMP7 as potential peripheral blood biomarkers in idiopathic pulmonary fibrosis. PLoS Med. 2008:5:e93.

12. Bauer $Y$, White ES, de Bernard S, Cornelisse P, Leconte I, Morganti A, et al. MMP-7 is a predictive biomarker of disease progression in patients with idiopathic pulmonary fibrosis. ERJ Open Res. 2017;3(1):00074-2016.

13. Richards TJ, Kaminski N, Baribaud F, Flavin S, Brodmerkel C, Horowitz D, et al. Peripheral blood proteins predict mortality in idiopathic pulmonary fibrosis. Am J Respir Crit Care Med. 2012;185(1):67-76.

14. O'Brien EC, Durheim MT, Gamerman V, Garfinkel S, Anstrom KJ, Palmer SM, et al. Rationale for and design of the idiopathic pulmonary fibrosisPRospective outcomes (IPF-PRO) registry. BMJ Open Respir Res. 2016;3(1): e000108.

15. Raghu G, Collard HR, Egan JJ, Martinez FJ, Behr J, Brown KK, et al. An official ATS/ERS/JRS/ALAT statement: idiopathic pulmonary fibrosis: evidence-based guidelines for diagnosis and management. Am J Respir Crit Care Med. 2011; 183(6):788-824.

16. Bhattacharya S, Dunham AA, Cornish MA, Christian VA, Ginsburg GS Tenenbaum JD, et al. The measurement to understand reclassification of disease of Cabarrus/Kannapolis (MURDOCK) study community registry and biorepository. Am J Transl Res. 2012;4(4):458-70.

17. Kuhn M. Building predictive models in R using the caret package. J Stat Softw. 2008;28(5):1-26.

18. Wells AU, Desai SR, Rubens MB, Goh NS, Cramer D, Nicholson AG, et al. Idiopathic pulmonary fibrosis: a composite physiologic index derived from disease extent observed by computed tomography. Am J Respir Crit Care Med. 2003;167(7):962-9.

19. Hankinson JL, Odencrantz JR, Fedan KB. Spirometric reference values from a sample of the general U.S. population. Am J Respir Crit Care Med. 1999; 159(1):179-87.

20. Crapo RO, Morris AH. Standardized single breath normal values for carbon monoxide diffusing capacity. Am Rev Respir Dis. 1981;123(2):185-9.

21. Finlay GA, Russell KJ, McMahon KJ, D'arcy EM, Masterson JB, FitzGerald MX, O'Connor CM. Elevated levels of matrix metalloproteinases in bronchoalveolar lavage fluid of emphysematous patients. Thorax. 1997;52(6):502-6.

22. Churg A, Zhou S, Wright JL. Series "matrix metalloproteinases in lung health and disease": matrix metalloproteinases in COPD. Eur Respir J. 2012;39(1): 197-209.

23. Hamai $\mathrm{K}$, Iwamoto $\mathrm{H}$, Ishikawa $\mathrm{N}$, Horimasu $\mathrm{Y}$, Masuda $T$, Miyamoto $\mathrm{S}$, et al. Comparative study of circulating MMP-7, CCL18, KL-6, SP-A, and SP-D as disease markers of idiopathic pulmonary fibrosis. Dis Markers. 2016;2016: 4759040 . 
24. Tzouvelekis A, Herazo-Maya JD, Slade M, Chu JH, Deiuliis G, Ryu C, et al. Validation of the prognostic value of MMP-7 in idiopathic pulmonary fibrosis. Respirology. 2017;22(3):486-93.

25. Raghu G, Richeldi L, Jagerschmidt A, Martin V, Subramaniam A, Ozoux ML, et al. Idiopathic pulmonary fibrosis. Prospective, case-controlled study of natural history and circulating biomarkers. Chest. 2018;154:1359-70.

26. Manoury B, Nenan S, Guenon I, Boichot E, Planquois JM, Bertrand CP, et al. Macrophage metalloelastase (MMP-12) deficiency does not alter bleomycininduced pulmonary fibrosis in mice. J Inflamm (Lond). 2006;3:2.

27. Matute-Bello G, Wurfel MM, Lee JS, Park DR, Frevert CW, Madtes DK, et al. Essential role of MMP-12 in Fas-induced lung fibrosis. Am J Respir Cell Mol Biol. 2007;37(2):210-21.

28. Flechsig P, Hartenstein B, Teurich S, Dadrich M, Hauser K, Abdollahi A, et al. Loss of matrix metalloproteinase-13 attenuates murine radiation-induced pulmonary fibrosis. Int J Radiat Oncol Biol Phys. 2010;77(2):582-90.

29. Sen Al, Shiomi T, Okada Y, D'Armiento JM. Deficiency of matrix metalloproteinase-13 increases inflammation after acute lung injury. Exp Lung Res. 2010;36(10):615-24.

30. Nkyimbeng T, Ruppert C, Shiomi T, Dahal B, Lang G, Seeger W, et al. Pivotal role of matrix metalloproteinase 13 in extracellular matrix turnover in idiopathic pulmonary fibrosis. PLoS One. 2013;8(9):e73279.

31. Craig VJ, Zhang L, Hagood JS, Owen CA. Matrix metalloproteinases as therapeutic targets for idiopathic pulmonary fibrosis. Am J Respir Cell Mol Biol. 2015;53(5):585-600.

32. Manetti M, Guiducci S, Romano E, Bellando-Randone S, Conforti ML, IbbaManneschi $L$, et al. Increased serum levels and tissue expression of matrix metalloproteinase-12 in patients with systemic sclerosis: correlation with severity of skin and pulmonary fibrosis and vascular damage. Ann Rheum Dis. 2012;71(6):1064-72.

33. Greene J, Wang M, Liu YE, Raymond LA, Rosen C, Shi YE. Molecular cloning and characterization of human tissue inhibitor of metalloproteinase 4. J Biol Chem. 1996:271(48):30375-80.

34. Tiede SL, Wassenberg M, Christ K, Schermuly RT, Seeger W, Grimminger F, et al. Biomarkers of tissue remodeling predict survival in patients with pulmonary hypertension. Int J Cardiol. 2016;223:821-6.

35. Yamashita CM, Dolgonos L, Zemans RL, Young SK, Robertson J, Briones N, et al. Matrix metalloproteinase 3 is a mediator of pulmonary fibrosis. Am J Pathol. 2011;179(4):1733-45.

36. Manicone AM, Huizar I, McGuire JK. Matrilysin (Matrix Metalloproteinase-7) regulates anti-inflammatory and antifibrotic pulmonary dendritic cells that express CD103 (alpha(E)beta(7)-integrin). Am J Pathol. 2009;175(6):2319-31.

37. Li Q, Park PW, Wilson CL, Parks WC. Matrilysin shedding of syndecan-1 regulates chemokine mobilization and transepithelial efflux of neutrophils in acute lung injury. Cell. 2002;111(5):635-46.

38. Belperio JA, Keane MP, Arenberg DA, Addison CL, Ehlert JE, Burdick MD, Strieter RM. CXC chemokines in angiogenesis. J Leukoc Biol. 2000;68(1):1-8.

39. Keane MP, Strieter RM, Lynch JP 3rd, Belperio JA. Inflammation and angiogenesis in fibrotic lung disease. Semin Respir Crit Care Med. 2006; 27(6):589-99.

40. Strieter RM, Belperio JA, Burdick MD, Keane MP. CXC chemokines in angiogenesis relevant to chronic fibroproliferation. Curr Drug Targets Inflamm Allergy. 2005;4(1):23-6.

41. Strieter RM, Belperio JA, Keane MP. CXC chemokines in angiogenesis related to pulmonary fibrosis. Chest. 2002;122(6 Suppl):298S-301S.

42. Strieter RM, Burdick MD, Gomperts BN, Belperio JA, Keane MP. CXC chemokines in angiogenesis. Cytokine Growth Factor Rev. 2005;16(6):593-609.

43. Garbacki N, Di Valentin E, Piette J, Cataldo D, Crahay C, Colige A. Matrix metalloproteinase 12 silencing: a therapeutic approach to treat pathological lung tissue remodeling? Pulm Pharmacol Ther. 2009;22(4):267-78.

44. Kang HR, Cho SJ, Lee CG, Homer RJ, Elias JA. Transforming growth factor (TGF)-beta1 stimulates pulmonary fibrosis and inflammation via a Baxdependent, bid-activated pathway that involves matrix metalloproteinase12. J Biol Chem. 2007;282(10):7723-32

45. Madala SK, Pesce JT, Ramalingam TR, Wilson MS, Minnicozzi S, Cheever AW, et al. Matrix metalloproteinase 12-deficiency augments extracellular matrix degrading metalloproteinases and attenuates IL-13-dependent fibrosis. J Immunol. 2010;184(7):3955-63.

46. Foronjy RF, Sun J, Lemaitre V, D'Armiento JM. Transgenic expression of matrix metalloproteinase- 1 inhibits myocardial fibrosis and prevents the transition to heart failure in a pressure overload mouse model. Hypertens Res. 2008;31(4):725-35.
47. limuro Y, Nishio T, Morimoto T, Nitta T, Stefanovic B, Choi SK, et al. Delivery of matrix metalloproteinase-1 attenuates established liver fibrosis in the rat. Gastroenterology. 2003;124(2):445-58.

48. Kaar JL, Li Y, Blair HC, Asche G, Koepsel RR, Huard J, Russell AJ. Matrix metalloproteinase-1 treatment of muscle fibrosis. Acta Biomater. 2008;4(5): 1411-20.

49. Radbill BD, Gupta R, Ramirez MC, DiFeo A, Martignetti JA, Alvarez CE, et al. Loss of matrix metalloproteinase-2 amplifies murine toxin-induced liver fibrosis by upregulating collagen I expression. Dig Dis Sci. 2011;56(2):406-16.

50. McQuibban GA, Gong JH, Wong JP, Wallace JL, Clark-Lewis I, Overall CM. Matrix metalloproteinase processing of monocyte chemoattractant proteins generates CC chemokine receptor antagonists with anti-inflammatory properties in vivo. Blood. 2002;100(4):1160-7.

51. Belperio JA, Keane MP, Burdick MD, Lynch JP 3rd, Xue YY, Berlin A, et al. Critical role for the chemokine MCP-1/CCR2 in the pathogenesis of bronchiolitis obliterans syndrome. J Clin Invest. 2001;108(4):547-56.

52. Moore BB, Kolodsick JE, Thannickal VJ, Cooke K, Moore TA, Hogaboam C, et al. CCR2-mediated recruitment of fibrocytes to the alveolar space after fibrotic injury. Am J Pathol. 2005;166(3):675-84.

53. Phillips RJ, Burdick MD, Hong K, Lutz MA, Murray LA, Xue YY, et al. Circulating fibrocytes traffic to the lungs in response to CXCL12 and mediate fibrosis. J Clin Invest. 2004;114(3):438-46.

54. Kim KH, Burkhart K, Chen P, Frevert CW, Randolph-Habecker J, Hackman RC, et al. Tissue inhibitor of metalloproteinase-1 deficiency amplifies acute lung injury in bleomycin-exposed mice. Am J Respir Cell Mol Biol. 2005;33(3): 271-9.

\section{Publisher's Note}

Springer Nature remains neutral with regard to jurisdictional claims in published maps and institutional affiliations.

Ready to submit your research? Choose BMC and benefit from:

- fast, convenient online submission

- thorough peer review by experienced researchers in your field

- rapid publication on acceptance

- support for research data, including large and complex data types

- gold Open Access which fosters wider collaboration and increased citations

- maximum visibility for your research: over $100 \mathrm{M}$ website views per year

At $\mathrm{BMC}$, research is always in progress.

Learn more biomedcentral.com/submissions 